\title{
PROBLEMA DA CATENÁRIA: HISTÓRIA, SOLUÇÃO E APLICAÇÕES
}

CATENARY PROBLEM: HISTORY, SOLUTION AND APPLICATIONS

\author{
Lucas Antonio Mendes de Lima ${ }^{1}$ \\ Sandra Regina Figueiredo de Miranda ${ }^{2}$
}

\section{Resumo}

O objetivo deste artigo é apresentar uma história sobre o Problema da Catenária, com sua solução e algumas aplicações. Foi abordada a etimologia da palavra catenária, o enunciado do problema foi proposto por Jakob Bernoulli, os matemáticos que contribuíram para sua solução, como Galileu, Huygens, Leibniz e Johann Bernoulli. É evidenciada a construção matemática da parábola e da catenária e uma corroboração desta distinção por meio de gráficos plotados no GeoGebra, seguida aplicações na engenharia e arquitetura. Nesse sentido, elucida-se a possibilidade de levar, para sala de aula, curiosidades deste tipo ao trabalhar com Função Quadrática para instigar os alunos sobre a existência de outras funções como as exponenciais e a possibilidade para desdobramento de pesquisas futuras com outros problemas ou situações presentes ao longo História da Matemática.

Palavras-chave: Educação Matemática. História da Matemática. Problema da Catenária.

\section{Abstract}

This paper presents a story about the catenary problem, with its solution and some applications. We approach the etymology of the catenary word, the statement of the problem proposed by Jakob Bernoulli, the mathematicians who contributed to his solution like Galileo, Huygens, Leibniz and Johann Bernoulli. Furthermore, we also emphasize the mathematical construction of the parable and catenary and a corroboration of this distinction through graphs plotted in GeoGebra, followed by applications in engineering and architecture. In this terms, we elucidate the possibility to taking curiosities of this sort into the classroom when working with Quadratic Function to instigate students about the existence of other functions such as exponentials and the possibility of unfolding future research with other actual problems or situations throughout the History of Mathematics.

Keywords: Mathematical Education. History of Mathematics. Catenary Problem.

\footnotetext{
${ }_{1}$ Membro Pesquisador do Grupo de Pesquisa em História, Educação e Matemática na Amazônia (GHEMAZ) da Universidade do Estado do Pará. Mestrando do Programa de Pós-Graduação em Ensino de Matemática da UEPA. E-mail: lucasamlima@outlook.com

${ }^{2}$ Mestranda do Programa de Pós-Graduação em Ensino de Matemática da Universidade do Estado do Pará E-mail: sandramir2014@gmail.com
} 
O auxílio da Educação Física na construção do saber matemático

\section{Introdução}

A proposta e motivação deste estudo tiveram início a partir da disciplina Ensino de Matemática I, cursada no Programa de Mestrado Profissional em Ensino de Matemática (PMPEM) vinculado à Universidade do Estado do Pará (UEPA). Consequentemente, a escolha do tema de pesquisa surgiu deste vínculo, visto que foi objeto de um estudo mais amplo da disciplina. Outro ponto que nos influenciou para o seu desenvolvimento foi a leitura do Livro "e: a história de um número", com o qual, encontramos, no capítulo 12, o problema da catenária proposto por um dos irmãos Bernoulli e que fez parte de um dos problemas mais instigantes para os matemáticos do século XVII.

Neste sentido, buscamos retomar este problema da História do Cálculo que envolveu uma indagação que gerou grande inquietude para a comunidade acadêmica do século XVII, o qual recaía na possibilidade de encontrar a equação representativa da catenária. Dessa maneira, matemáticos do período buscaram encontrar sua solução, dentre eles, Galileu, ao considerar como hipótese provar que a curva até então denominada por catenária era uma parábola.

A partir destas motivações, evidenciamos que o objetivo desta da pesquisa realizada foi apresentar uma história sobre o Problema da Catenária, com sua solução frente às contribuições dos estudiosos da época, na linguagem atual da Matemática e algumas aplicações em outros ramos da Ciência. Para alcançarmos este objetivo, recorremos à pesquisa bibliográfica, a qual, de acordo com Gil (2008), é fundamentada em trabalhos acadêmicos como pesquisas em nivel de teses, dissertações, artigos e livros científicos com a presença da temática por nós abordada.

No decorrer do artigo, abordaremos a etimologia da palavra catenária; o problema na íntegra que foi proposto por Jakob Bernoulli;, sua solução e os matemáticos que contribuíram para ela; sua relação com a trigonometria hiperbólica; a distinção geométrica entre parábola e catenária e algumas aplicações desta na arquitetura e na engenharia. 
O auxílio da Educação Física na construção do saber matemático

\section{Afinal o que é uma catenária? Em que consiste este problema?}

Entre os problemas de destaque, nas décadas que se seguiram à invenção do Cálculo, estava o da catenária, palavra que se caracteriza etimologicamente por uma corrente suspensa (do latim catena, corrente), segundo Maor (2008). Neste sentido, a fim de caracterizarmos tal problema, abordamos, a seguir, uma breve história do seu surgimento, por quem ele foi proposto, consequentemente enunciado, assim como, quais os estudiosos que contribuíram para apresentar sua solução.

De acordo com Maor (2008), o problema foi proposto inicialmente por um dos irmãos Bernoulli, dessa vez Jakob. No ano de 1690 do Acta eruditorum, o jornal que Leibniz havia fundado oito anos antes, Jakob escreveu o seguinte enunciado:

"E agora vamos propor este problema: encontrar a curva formada por um fio pendente, livremente suspenso a partir de dois pontos fixos". Jakob presumiu que o fio é flexivel em todas as suas partes e que tem uma espessura constante (e, portanto, uma densidade linear uniforme). (MAOR, 2008, p. 183).

O problema proposto por Jakob pode ser mais bem expresso visualmente por uma imagem que, de certa maneira, é comum em algumas cidades, como evidenciamos, a seguir, na figura 1 .

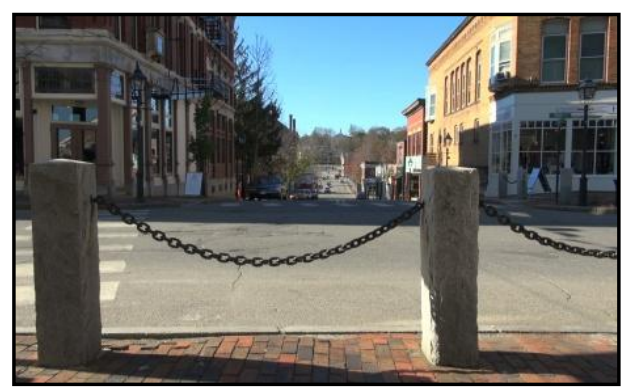

Figura 1: exemplo de uma catenária

Fonte: http://hotsite.tvescola.org.br/matematica

Segundo Maor (2008), Galileu havia demonstrado interesse em resolvê-lo e imaginava que a curva era uma parábola. Mas, Chrístian Huygens, o prolífico cientista holandês, provou que a catenária não podia ser 
uma parábola, embora ele não tenha encontrado a curva correta que a representasse.

De acordo com Coelho (2008), em junho de 1691, um ano depois de Jakob Bernoulli ter proposto o seu problema, o jornal Acta eruditorum publicou as três soluções, apresentadas por Huygens, Leibniz e Johann Bernoulli. Cada uma delas abordava o problema de uma maneira, mas todos chegaram à mesma solução. Johann acrescentou que, das duas curvas, a parábola é algébrica enquanto a catenária é transcendental.

Segundo Faria (2011), a demonstração de Johann Bernoulli era mais simples que a de Leibniz, a solução consistia em uma dedução a partir de argumentação da mecânica clássica dos corpos em equilíbrio, uma equação diferencial que deveria ser satisfeita pela curva, determinando a equação diferencial abaixo:

$$
\frac{d y}{d x}=\frac{a}{s}
$$

sendo a uma constante.

Temos que:

$$
s=\int \sqrt{\left[1+\left(\frac{d x}{d y}\right)^{2}\right]} d y
$$

$X$ e $y$ aparecem implicitamente em s, por esse motivo a equação diferencial não pode ser resolvida diretamente.

Por este motivo, Bernoulli transforma a equação diferencial

$$
\frac{d y}{d x}=\frac{a}{s}
$$

em uma equação diferencial que envolve $x$ e $y$ explicitamente, obtendo:

$$
d y=\frac{a d x}{\sqrt{\left(x^{2}-a^{2}\right)}}
$$


A resolução desta equação determina a curva que a satisfaz.

De acordo com Coelho (2018), a demonstração de Bernoulli, trouxe grandes contribuições ao desenvolvimento do Cálculo, mais tarde conhecido como Cálculo de Variações.

Apresentamos, também, uma construção desenvolvida por Leibniz em 1960, a qual também foi evidenciada por Maor (2008), na figura 2, com $\mathrm{a}=1$.

$$
f(x)=\frac{\left(e^{x}+e^{-x}\right)}{2}
$$

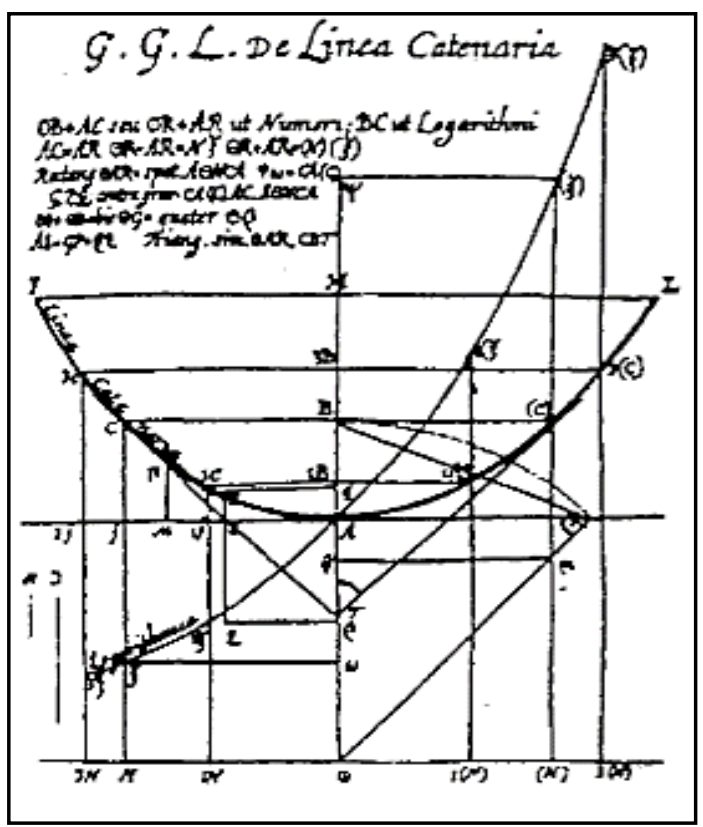

Figura 2 - Construção de Leibniz (1960) Fonte: Maor (2008, p. 186)

Para além da função abordada anteriormente, Maor (2008), também ressalta uma segunda lei de formação, representada pela relação abaixo, seguida de sua construção gráfica (figura 3). 


$$
f(x)=\frac{\left(e^{x}-e^{-x}\right)}{2}
$$

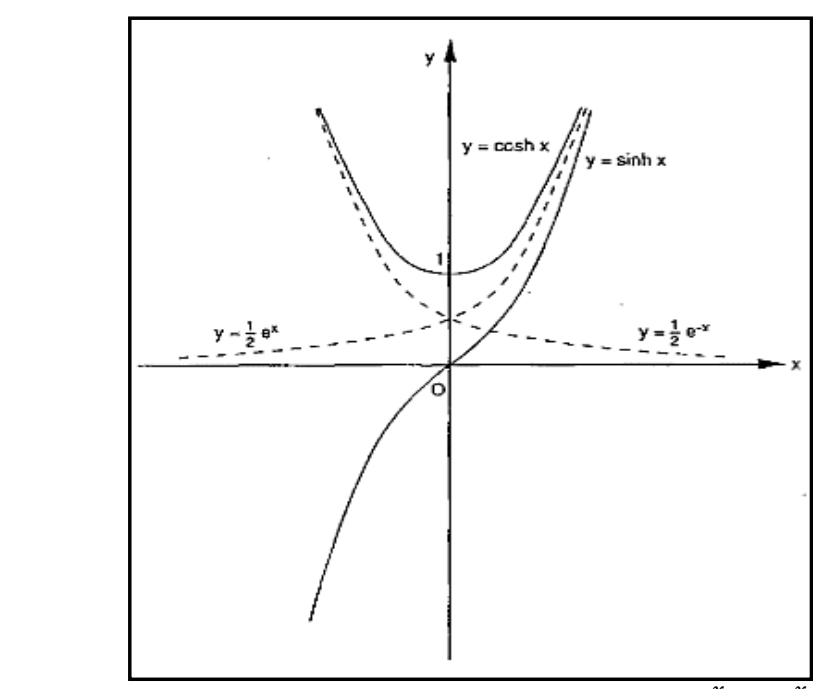

Figura 3 - Gráfico da função $f(x)=\frac{\left(e^{x}-e^{-x}\right)}{2}$

Fonte: Maor (2003, p. 188)

A partir destas duas funções, podem ser observadas semelhanças com as funções circulares $\cos (x)$ e $\operatorname{sen}(x)$ estudadas em trigonometria. Notações que foram introduzidas por Vincenzo Riccati $(1707$ - 1775), representadas por Ch $x$ e $S h x$ e as representou, respectivamente, por:

$$
\operatorname{Ch} x=\frac{\left(e^{x}+e^{-x}\right)}{2} \quad \operatorname{Sh} x=\frac{\left(e^{x}+e^{-x}\right)}{2}
$$

Riccati demonstrou que as funções expressas anteriormente satisfazem a identidade $(\operatorname{Ch} \varphi)^{2}-(\operatorname{Sh} \varphi)^{2}=1$ (a letra $\varphi$ simboliza a variável independente), a qual, exceto pelo sinal de menos no segundo termo, é análoga a identidade trigonométrica $(\operatorname{Cos} \varphi)^{2}+(\operatorname{Sen} \varphi)^{2}=1$. Essa identidade indica que $\operatorname{Ch} \varphi$ e $\operatorname{Sh} \varphi$ (p estão relacionados com a hipérbole $x^{2}-y^{2}=1$, do mesmo modo $\operatorname{como} \operatorname{Cos} \varphi$ e $\operatorname{Sen} \varphi$ se relacionam com o círculo unitário $x^{2}+y^{2}=1$. A notação de Riccati sobreviveu quase inalterada. Hoje, chamamos essas funções de $\operatorname{Cosh} \varphi$ e $\operatorname{Senh} \varphi$ que possuem por significado “cosseno hiperbólico de $\varphi$ " e "seno hiperbólico de $\varphi$ ".

Trabalhos atuais apontam que a maioria das identidades trigonométricas possuem equivalentes hiperbólicos. Basta que sejam considerados, para isso, o seno e cosseno hiperbólico ao invés dos convencionais. 
A partir das relações supracitadas, apresentamos, a seguir, a distinção entre parábola e catenária, como elas são compostas matematicamente e geometricamente por meio do software GeoGebra.

\section{Parábola e Catenária}

A partir desse breve relato histórico, apresentamos a construção matemática da parábola e da catenária, com a finalidade de compreendermos sua composição e, consequentemente, observarmos a distinção entre elas. Vale ressaltar que, a primeira, é normalmente trabalhada nos cursos de Geometria Analítica, enquanto, a segunda, é estudada nos cursos de Cálculo avançado.

\section{Parábola}

Dados um ponto $\mathrm{F}$ e uma reta $\mathrm{d}$, pertencentes a um plano $\square$, com $F \notin d$, seja $\mathrm{p}$ a distância entre $\mathrm{F}$ e d. Parábola é o conjunto de pontos de a que estão à mesma distância de F e de d. Iezzi (2005).

$$
\text { Parábola }=\{P \in \alpha \text { tq } P F=P d\}
$$

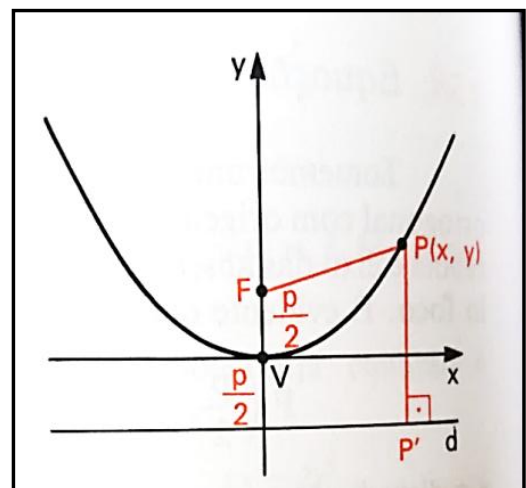

Figura 4 - Gráfico da parábola

Fonte: Iezzi (2005, p. 178)

Pela definição, conclui-se que:

$$
\mathrm{PF}=\mathrm{PP}
$$




$$
\sqrt{(x-0)+\left(y-\frac{p}{2}\right)^{2}=\sqrt{(x-x)^{2}+\left(y-\frac{p}{2}\right)^{2}}}
$$

De onde, obtém-se:

$$
x^{2}=2 p y
$$

\section{Catenária}

A notação moderna $y=\frac{\left(e^{a x}+e^{-a x}\right)}{2 a}$ da curva Catenária, também pode ser escrita na forma $y=\frac{1}{2 a}\left(e^{a x}+e^{-a x}\right)$ cujo gráfico está representado na figura 5. A demonstração pode ser verificada na obra de Simmons (1987, p. 611).

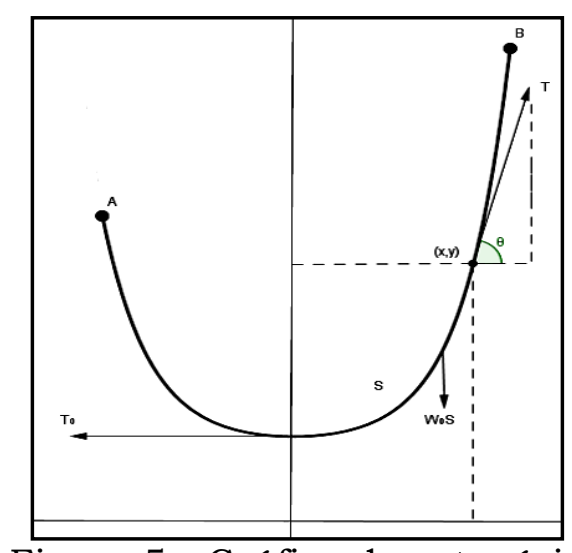

Figura 5 - Gráfico da catenária

Fonte: Simmons (1987, p. 611)

Pode-se obter a diferenciação da catenária quando a parte da corrente entre o ponto mais baixo e $(\mathrm{x}, \mathrm{y})$ está em equilíbrio estático sob a ação de três forças: a tensão no ponto mais baixo, a tensão variável $T_{0} \mathrm{em}$ $(\mathrm{x}, \mathrm{y})$ que age na direção da tangente devido à flexibilidade do fio e uma força para baixo $W_{0} s$ igual ao peso do fio entre esses pontos. Sejam $s$ o comprimento do arco, entre esse ponto e um ponto variável (x,y), e $W_{0}$ a densidade linear (peso por unidade de comprimento) do fio. Igualando o 
membro horizontal de $\mathrm{T}$ a $T_{0}$ e o membro vertical de $\mathrm{T}$ ao peso da corrente, temos:

$$
T \cos \theta=T_{0} \text { e T } \operatorname{sen} \theta=W_{0} S
$$

Dividindo um pelo outro, o T é eliminado e obtém-se

que é igual a:

$$
\operatorname{tg} \theta=\frac{W_{0} s}{T_{0}}
$$

$$
\frac{d y}{d x}=\text { as, onde } a=\frac{W_{0}}{T_{0}}
$$

Assim, eliminando a variável s e derivando em relação à $\mathrm{x}$, temos:

$$
\frac{d^{2} y}{d x^{2}}=a \frac{d s}{d x}-a \sqrt{1+\left(\frac{d y}{d x}\right)^{2}}
$$

A equação (1) encontrada é a equação diferencial da catenária. Determinando, agora, a equação (1) por integrações sucessivas. Esse procedimento é promovido pela entrada da variável auxiliar

$$
p=\frac{d y}{d x}
$$

Substituindo-se em (1) teremos,

$$
\frac{d p}{d x}=a \sqrt{1+p^{2}}
$$

separando as variáveis e integrando ambos os membros, obtemos a equação abaixo:

$$
\int \frac{d_{p}}{\sqrt{1+p^{2}}}=\int a d x
$$


Substituindo $p=\operatorname{tg} \theta$ no primeiro membro da igualdade, obtemos

Assim,

$$
d p=\sec \emptyset d \emptyset \mathrm{e} \sqrt{1+p^{2}}=\sec \emptyset .
$$

$$
\int \frac{d_{p}}{\sqrt{1+p^{2}}}=\int \frac{d_{p}}{\sqrt{1+p^{2}}}=\int \sec \emptyset d \emptyset=\ln (\sec \emptyset+\operatorname{tg} \emptyset)=\ln \left(\sqrt{1+p^{2}}+p\right)
$$

Logo, a equação (2) ficará igual a:

$$
\left(\sqrt{1+p^{2}}+p\right)=a x+c_{1}
$$

Se $x=0, \operatorname{logo} p=0$, portanto $c_{1}=0$.

Assim,

$$
\left(\sqrt{1+p^{2}}+p\right)=a x
$$

Resolvendo a equação em p, teremos:

$$
\frac{d y}{d x}=p=\frac{1}{2}\left(e^{a x}+e^{-a x}\right)
$$

Integrando ambos os membros:

$$
y=\frac{1}{2 a}\left(e^{a x}+e^{-a x}\right)+c_{2} .
$$

Se colocarmos equação anterior na origem do sistema de coordenadas no ponto $\left(0, \frac{1}{a}\right)$, como mostra a figura 1 , a equação adota sua forma final,

$$
y=\frac{1}{2 a}\left(e^{a x}+e^{-a x}\right)
$$

A equação (3) revela a natureza matemática precisa da catenária e pode ser usada como base para posteriores investigações de suas propriedades. 
Segundo Maor (2004), a descoberta da equação da catenária foi um grande triunfo do novo Cálculo Diferencial. A notação moderna da catenária demonstrada em (3), onde aé uma constante cujo valor depende dos parâmetros físicos da corrente - sua densidade linear (massa por unidade de comprimento) e a tensão com a qual ela é segura.

\section{Parábola e catenária: graficamente}

A partir da construção matemática evidenciada anteriormente, com a atualização do software GeoGebra, ressaltamos individualmente o formado geométrico da parábola, da catenária, assim como, de ambas para corroborar tal distinção gráfica (figura 6).

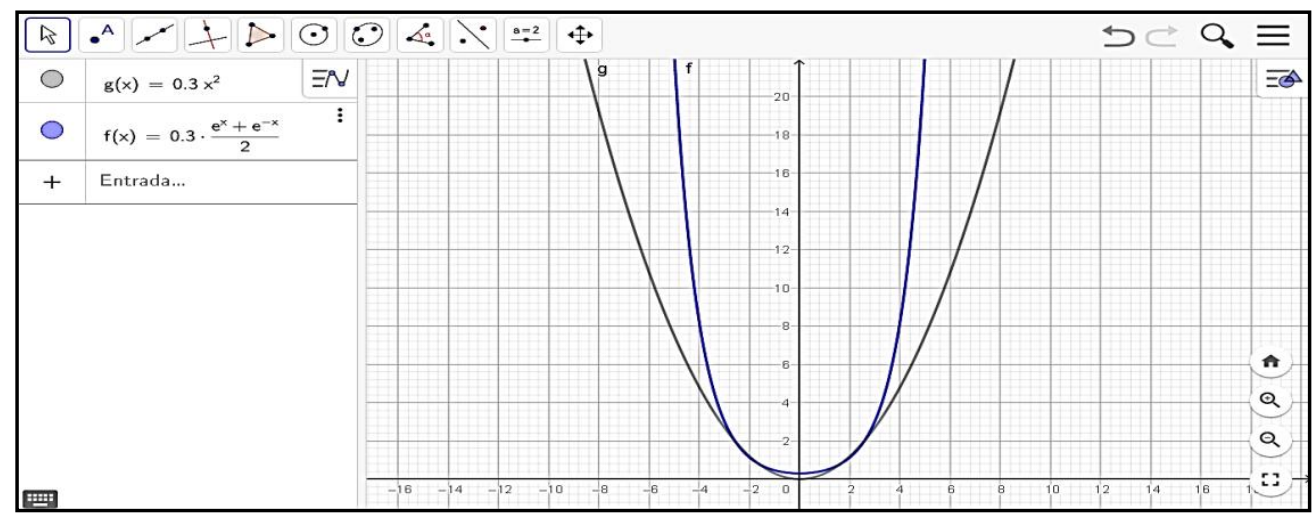

Figura 6 - Distinção entre parábola e catenária no GeoGebra Fonte: Elaborada pelos autores

Diante da abordagem desenvolvida, fica evidente tal distinção entre as representações da parábola e da catenária, tanto algébrica, quanto geometricamente evidenciada pelo GeoGebra. A partir disso, mostraremos a seguir algumas aplicações da catenária em áreas como a arquitetura e a engenharia.

\section{Aplicações da catenária:}

Neste âmbito, abordamos algumas aplicações da catenária em outros ramos da ciência que estão para além da Matemática. Com ênfase 
especificamente para a arquitetura e para engenharia frente às obras de personagens reconhecidos internacionalmente como Eero Saarinen e Antonio Gaudí.

\section{Eero Saarinen (1910 - 1961)}

Arquiteto e designer finlandês migrou para os EUA em 1923. Em 1947, venceu o concurso para o Gateway Arc, em Sant Louis, Missouri. A obra foi concebida como um enorme arco localizado às margens do Rio designado por Mississipi. O arco é uma curva catenária, cujo vão e altura possuem ambos, 192 metros. Ele consiste em uma dupla pele de aço - na parte externa, aço inoxidável e, na parte interna aço carbono (figura 7).

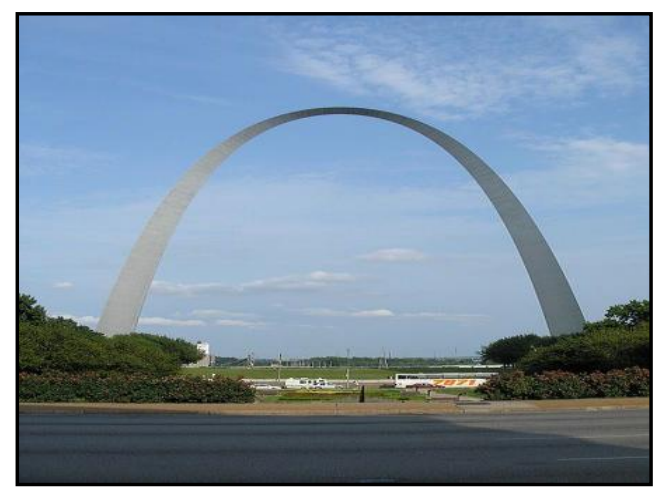

Figura 7 - Arco do Portal em St. Louis

Fonte: https://naotrivial.wordpress.com

\section{Antonio Gaudi (1852 - 1926)}

Arquiteto espanhol nascido na Catalunha, Espanha, segundo ORCIUOLI (2002), Gaudí incorporou definitivamente ao seu repertório o uso de catenárias por razões estruturais. Entre todos os arcos, ele caracterizou a catenária como a mais mecânica, uma vez que a linha de pressão segue exatamente a forma do arco, como podemos observar nas figuras 8 e 9 que ilustram a sua construção. 


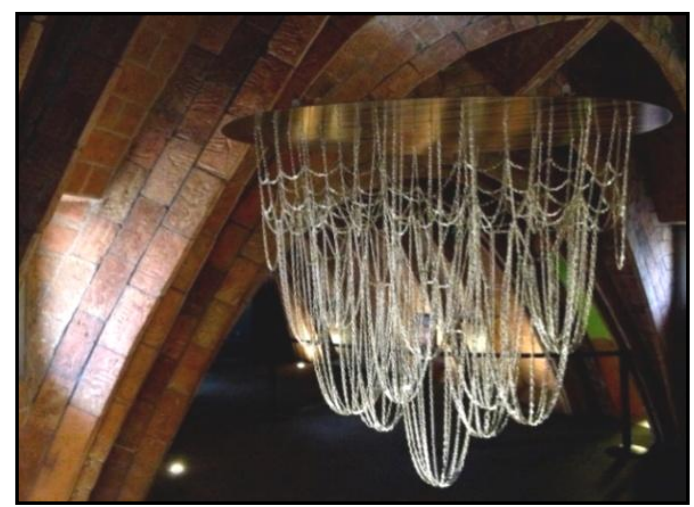

Figura 8 - Correntes de Gaudi, na Casa Milà, Barcelona, Espanha Fonte: https://naotrivial.wordpress.com

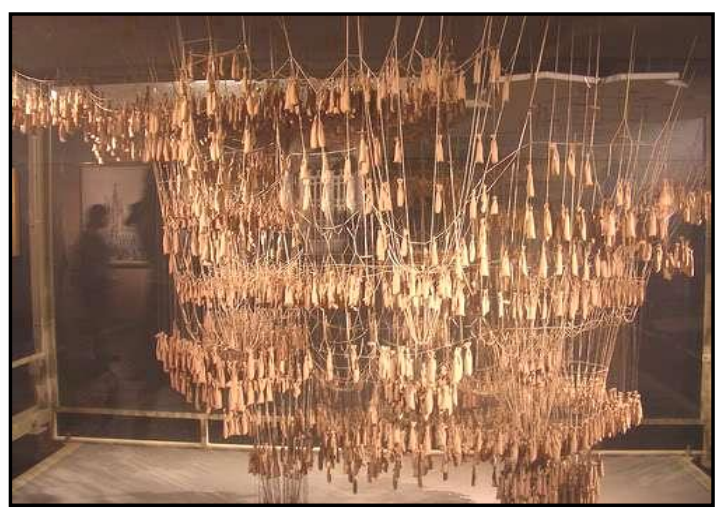

Figura 9 - Obra de Gaudí - Pesos deformando as catenárias Fonte: https:/ / naotrivial.wordpress.com

A partir do exposto, a considerar desde o contexto histórico, apresentado neste artigo, observamos que um dos irmãos Bernoulli, Jakob, quando propôs o desafio, possivelmente estava preocupado com o desenvolvimento da Matemática enquanto Ciência. Porém, seu desenvolvimento não era tão simples para o aparato acadêmico do século XVII - isto gerou uma comoção pela comunidade acadêmica da época.

Nesse sentido, podemos perceber que a sua construção matemática além de desafiadora, possibilitou estudos que transcenderam o Cálculo como é o caso das obras supracitadas, fato que caracteriza a relevância da temática para a Ciência. Com isto, foram estabelecidas algumas con- 
O auxílio da Educação Física na construção do saber matemático

siderações acerca de tal relevância para a ciência, academia e educação básica.

\section{Considerações Finais}

Diante do objetivo deste artigo, que foi retomar o problema da catenária presente na História do Cálculo, frente às contribuições dos estudiosos da época e algumas aplicações em outros ramos da ciência, destacamos como fator preponderante para seu desenvolvimento, a História da Matemática, enquanto ferramenta para compreensão e esclarecimentos dos fatos, assim como para evidenciar os personagens que fizeram parte desta história. Nesse sentido, elucidamos as principais contribuições que estes estudiosos desencadearam para solucionar o problema do século XVII. Assim como desconstruções e distinções entre as curvas catenária e parábola; além da dimensão que os estudos da época proporcionaram à sociedade atual, como intencionamos apresentar, com as aplicações no ramo da arquitetura, engenharia, nas correntes de proteção das calçadas entre outras utilidades.

Outro ponto que pode ser ressaltado é a possibilidade de levar para sala de aula curiosidades deste tipo ao trabalhar com Função Quadrática, para instigar os alunos sobre a existência e distinção desta com outras funções como as exponenciais - igualmente, no estudo de Trigonometria, ao sugerir como possibilidade de abordagem, para este conteúdo, a Trigonometria Hiperbólica.

Por fim, ressaltamos que estudos como este estabelecem possibilidades para desdobramento de pesquisas futuras com outros problemas do gênero ou mesmo em situações presentes ao longo da História e da construção dos conteúdos matemáticos.

\section{Referências}

Matemática \& Ciência, v. 4, n. 1, p. 37-51, jun. 2021 - ISSN 2674-9416 
ALBUQUERQUE, Yuri; MIRANDA, Sarah. Tráctriz e Catenária: modelos e métodos matemáticos. São Paulo: Unicamp, 2015.

Disponível em:

https://www.ime.unicamp.br/ rmiranda/wordpress/wpcontent/uploads/2015/12/Tractriz.pdf. Acesso em: 27 jun. 2019.

CHING, Francis D. K. JARZOMBECK, M. M. PRAKASA, V. História Global da Arquitetura. São Paulo: Editora Senac, 2016.

COELHO, Rejeane Alexandre. A história dos problemas da tautócrona e da braquistócrona. Dissertação (Mestrado em Educação Matemática) - Universidade Estadual Paulista, Rio Claro, 2008.

FARIA, Sirlene Resende. A Catenária. Monografia (Especialização em Matemática) - Universidade Federal de Minas Gerais, 2011.

IEZZI, Gelson. Fundamentos de Matemática Elementar 7: Geometria Analítica. 5. ed. São Paulo: Atual, 2005.

MAOR, Eli. e: a história de um número. Tradução de Jorge Calife. 5. ed. Rio de Janeiro: Record, 2008.

ORCIUOLI, Affonso. Gaudí 150 anos - Documento. Revista AU, a. 17, n. 104 out./nov. 2002.

SIMMONS, George F. Cálculo com Geometria Analítica. Tradução de Seiji Hariki. São Paulo: Pearson Makron Books, 1987. v. 1.

\section{Sites Consultados:}

VILLAR, Fernando. A arte de construir pontes. Dica do professor.

Disponível em: http://hotsite.tvescola.org.br/matematica-em-toda-parte-

/fasciculos/transporte/. Acesso em: 30 maio 2019.

GNOG, Gustavo. A Catenária, Gaudí e Confusões de Nomenclatura. Não trivial. Disponível em: https://naotrivial.wordpress.com/2017/03/29/a-catenaria-gaudi-e-confusoes-denomenclatura/. Acesso em: 30 maio 2019.

Artigo recebido em: 19 abr. 2021

Artigo aprovado em: 28 jun. 2021 